\title{
Therapeutic Value of Using Two-step Splinting for Mallet Finger
}

\author{
Kazuo Saito ${ }^{1}$, Hitoshi Kihara, MD $^{2}$ \\ ${ }^{1}$ Department of Rehabilitation, Fuchinobe General Hospital \\ ${ }^{2}$ Kihara Orthopaedic Clinic
}

\begin{abstract}
In the present study, we report two cases of splinting therapy where favorable results were obtained using splinting of two types, consisting of a first step of fixation at PIP joint extension position and DIP joint extension position, followed by a second step of further fixation at DIP joint extension position, followed thereafter by hand therapy on mallet finger. As the first splint flexes the PIP joint, and, further, as the DIP joint is taken as the hyperextension position, it is thought that the ruptured tendon ends sufficiently entwined more than with the conventional splint at the DIP joint extension position. The splinting therapy we used, with its two-type splinting, is thought to have enabled fixation and ROM acquisition that were in tandem with the tendon healing process. This treatment hints at the effectiveness of conservative therapy in the treatment of swan neck deformities and bony mallet finger.
\end{abstract}

Keywords: tendinous mallet finger, bony mallet finger, two-step splinting, hand therapy

(Asian J Occup Ther 12: 23-28, 2016)

\section{Introduction}

Generally, conservative therapy is used to treat mallet finger. For simple closed mallet finger, surgery is controversial because results of studies indicate that splinting is effective. Nevertheless, surgery is indicated under certain conditions $[1,2]$. However, difficulties often arise in such cases because of extension lag. Thus, fixing a splint with slight extension at the distal interphalangeal (DIP) joint is often used in conservative treatment [2-6]. The standard recommendation is splinting for $\geq 6$ weeks, followed by active movement $[2,4]$. The splinting method has not been standardized, and various techniques have been reported. Research on specific fixing methods used in conservative treatment of mallet finger includes studies on the effectiveness of fixing the proximal interphalangeal (PIP) and DIP joints by Wehbé and Schneider [7], Evans [8], and O'Conner [9], but their studies did not include comparisons of the immobilization duration or types of splints. Pike et al.

Received: 18 April 2014, Accepted: 20 February 2015

Corresponding to: Kazuo Saito, Department of Rehabilitation, Fuchinobe General Hospital, 3-2-8 Fuchinobe, Chuouku, Sagamihara-City, Kanagawa, 252-0206, Japan

e-mail: kasuo_saitoh@yahoo.co.jp

C2016 Japanese Association of Occupational Therapists
[10] and O'Brien and Bailey [11] compared three types of splints, and both studies concluded that custom thermoplastic splints, which are custom-made for each patient, were most effective.

Considering that sufficient improvement of extension lag has not been obtained through splinting of the DIP joint alone, we believe that improved results could be observed by studying splinting of the PIP joint [7-9] and splinting duration. We believe so because we thought that while splinting the PIP and DIP joints does sufficiently draw together the ruptured tendon portions and that this would be a favorable position for repair of the terminal tendon, long-term immobilization would result in PIP joint contracture. Thus, to minimize PIP joint contracture, we thought that if we removed only the PIP joint splint at 2 to 3 weeks and then retained only the DIP splint, we could then correct the DIP joint extension lag.

We also believe that the key to the success of this approach would be having a hand therapist create a custom-made thermoplastic splint for each patient.

The present study aimed to determine the therapeutic values of two methods of splinting that involved initial fixation at the PIP and DIP joint extension positions, followed by further fixation at the DIP joint extension position, and then by hand therapy. Herein, we report splint therapy cases in which favorable results were 
obtained by using the two methods of splinting. This study was conducted after providing sufficient explanations to the subjects and obtaining their consent.

\section{Methods}

In this paper, we describe the splint therapy that we designed and the follow-up hand therapy, and report 2 cases, 1 each of tendinous and bony mallet fingers.

The protocols for the two-step splinting and hand therapy were as follows (Fig. 1):

\section{1) Two-step splinting (Table 1)}

Step 1 was performed up to 2 or 3 weeks after injury; it involved creating the splint for DIP joint hyperextension and $45^{\circ}$-flexion position of the PIP joint. The splint material used was a 1.6-mm Orfit soft-type material (Orfit Industries n.v. Belgium) and the splint was created with a full-circumference mold. Then, 2- or 3 -week fixation was performed (initial splinting).

Step 2 was performed from 2 or 3 weeks to 6 weeks; after 3 weeks' fixation with the first splint, we created a splint for the DIP joint slight extension position. By using a 1.6-mm Orfit soft-type material, a figure-8 splint was created. Here, 3-week fixation (second splint) was performed (total splint-fixation duration: 6 weeks). Subsequently, a DIP joint (night splint) was used for 12 weeks, with constant monitoring of the subject's condition. During this period, active range of motion (ROM) was initiated, but because the extension lag of the DIP joint became aggravated, splint use was increased from nighttime only to 24 hours [2, 4] (Fig. 1).

\section{2) Hand therapy}

Six weeks from the initiation of therapy, active exercise was initiated at the rehabilitation clinic. In cases

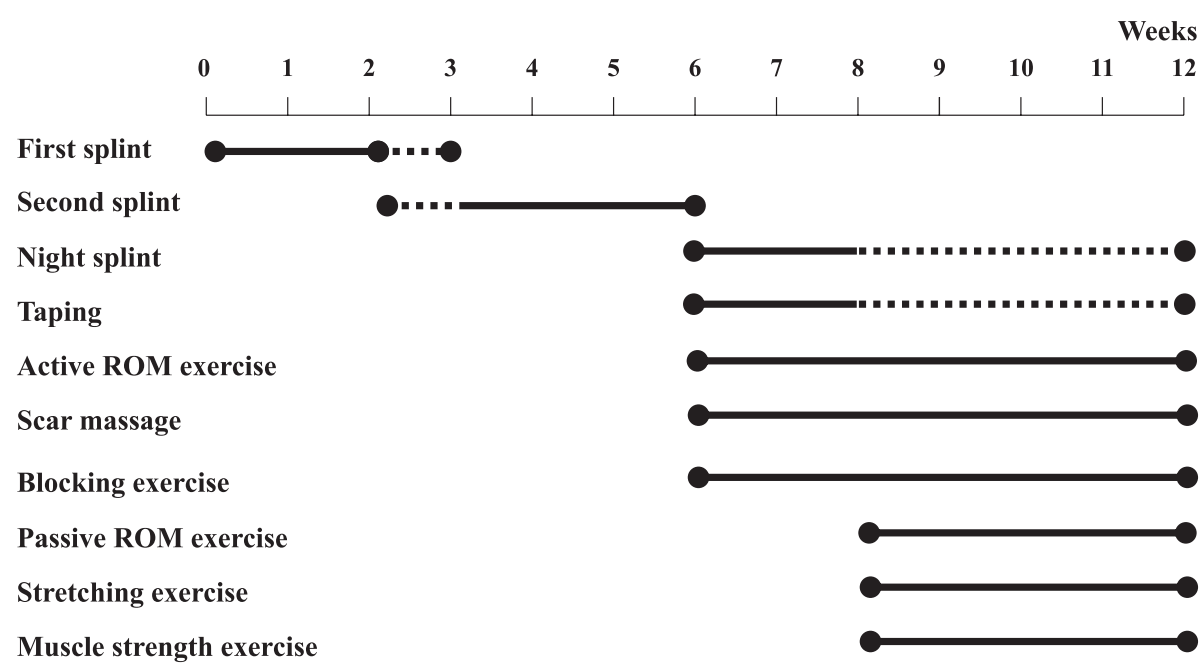

Fig. 1. Two-step splinting protocol and hand therapy.

Table 1. Two-step Splinting.

\begin{tabular}{|c|c|c|}
\hline & First step & Second step \\
\hline \multicolumn{3}{|l|}{ Appearance } \\
\hline Position & $\begin{array}{l}\text { DIP joint mild extended position } \\
\text { PIP joint flexion } 30-45^{\circ}\end{array}$ & DIP joint mild extended position \\
\hline Splint type & Circumference type & Eight figure type \\
\hline Material thickness & $1.6 \mathrm{~mm}$ & $1.6 \mathrm{~mm}$ \\
\hline \multirow{2}{*}{ Term } & 2 or 3 weeks from initial visit & 3 or 4 weeks after the first step splint \\
\hline & \multicolumn{2}{|c|}{ Total 6 weeks } \\
\hline Mounting time & Throughout the day & Throughout the day \\
\hline
\end{tabular}


where swelling remained on the dorsal side of the DIP joint, scar massage was performed from the proximal to the distal area, after the swelling was reduced. DIP joint blocking training was performed. The patient wore the splint in the time when there was no training (Table 2). Eight weeks from the initiation of therapy, other movement exercises and stretching were added. Muscular strengthening exercises of the extensor tendon were performed, with gradual increase of exercises. Muscular strength training of the extensor tendon consisted of gradually increasing the time and weight of pinch exercises, and extension resistance exercises, along with the use of Ceraplast. At first, the resistance time was short and the load was light, and the subject was monitored for pain and fatigue when the soft-type Ceraplast was used. Harder materials were gradually introduced (Table 2). Taping was performed mainly on the dorsal side of the DIP joint in a figure-8 configuration to offer support to prevent hyperflexion of the joint (Table 2). Taping was also instructed if the patient intended to the finger ends during the daytime. The areas indicated by the dotted line are sites subject to consultation with the physician during monitoring of the improvement of the extension lag (Fig. 1).

\section{Case Report}

Case 1 was a severe DIP joint flexion deformity due to tendinous mallet finger and a swan-neck deformity. Case 2 was a bony mallet finger that was treated with conservative therapy.

Case 1 was observed in a woman aged 27 years.
Her left little finger was injured by a tape cutter that had fallen from a high place. Two days thereafter, she was examined at our hospital and diagnosed with a tendinous mallet finger on radiography. She had no notable medical history. She presented a swan-neck deformity at the initial examination (Fig. 2).

On day 2 after the injury, the first splint was created and was affixed for 3 weeks. Thereafter, the second splint was created and was affixed for 3 weeks (total splint-setting period: 6 weeks), and ROM training consisting of DIP joint active movement was initiated. Scar massage was performed, and at 8 weeks after injury, other movement exercises, stretching, and muscular strength training were initiated. The DIP joint ROM over time in terms of extension/flexion was as follows: at the time of injury, $-60^{\circ} / 60^{\circ}$; 6 weeks after injury, $10^{\circ} / 30^{\circ}$; and 12 weeks after injury, $-5^{\circ} / 60^{\circ}$ with pain within manageable bounds. The patient was able to return to her part-time job at a convenience store. Her condition was classified as good according to the Crawford evaluation criteria [11]; the results are shown in Table 3 and Fig. 2.

Case 2 was observed in a man aged 28 years. His right ring finger was injured when it became stuck in a refrigerator while working. Three days thereafter, he was examined at our hospital and diagnosed with a mallet fracture on radiography. According to the Wehbé and Schneider classification system [7], the injury was classified as a type IIB injury. The patient had no notable medical history (Fig. 3).

On day 6 after injury, the first splint was created and worn for 2 weeks. Thereafter, the second splint was

Table 2. Hand therapy for 6 weeks and 8 weeks (ring finger).

1. Active ROM exercise
2. Blocking exercise
$\begin{aligned} & \text { 2. Scar massage } \\ & \text { Proximal } \rightarrow \text { distal }\end{aligned}$
$\begin{aligned} & \text { 3. Taping } \\ & \text { During the daytime } \\ & \text { at work }\end{aligned}$

Direction of the arrow indicates the movement of the injured finger 
Table 3. Case 1: Course of treatment (left little finger).

\begin{tabular}{lccccc}
\hline & 1 week & 2 weeks & 6 weeks & 12 weeks & Outcome \\
\hline DIP Ex/Flex & $-50 / 60$ & $20 /-20$ & $10 / 30$ & $-5 / 60$ & Crawford \\
Pain VAS & $7 / 10$ & $3 / 10$ & $1 / 10$ & $0 / 10$ & evaluation criteria \\
Work & Leave of absence & Some return & Some return & Full return & Good \\
\hline
\end{tabular}

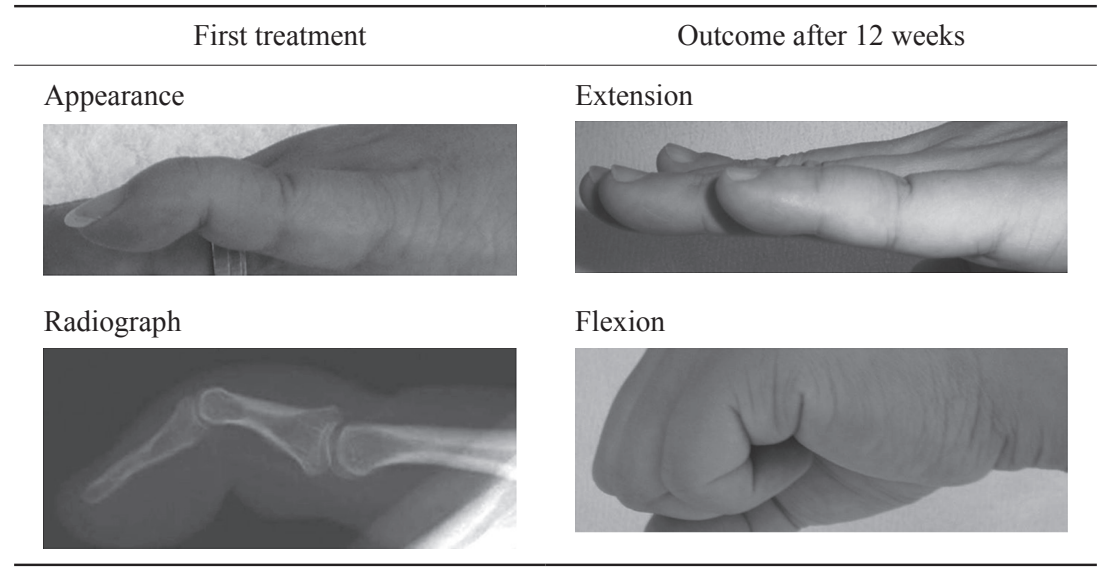

Fig. 2. Case 1: First treatment and outcome after 12 weeks (Left little finger with tendinous mallet finger).

created and worn for 4 weeks (total splint-setting period: 6 weeks), and ROM training via DIP joint autonomous movement was initiated. Scar massage was performed, and at 8 weeks after injury, other movement exercises, stretching, and muscular strength training were initiated. The DIP joint ROM over time in terms of extension/ flexion was as follows: at the time of injury, $-40^{\circ} / 50^{\circ}$; 6 weeks after injury, $20^{\circ} / 30^{\circ}$; and 12 weeks after injury, $5^{\circ} / 50^{\circ}$, with pain within manageable bounds and no pain during carrying tasks. This injury was classified as perfect according to the Crawford evaluation criteria [12]; the results are shown in Table 4 and Fig. 3.

\section{Discussion}

Anatomically, the balance between the central slip of the extensor hood at the PIP joint and the terminal extensor tendon at the DIP joint is important for finger function and appearance. Loss of the terminal extensor tendon attachment leads to the central slip receiving all tension. The PIP joint will thus have a resting tone in extension or even in hyperextension. With the flexed posture of the DIP joint, this would present as a swanneck deformity $[4,12,13]$. In mallet finger, the extent of the DIP joint flexion deformity is thought to be proportional to the extent of the separation of the ruptured tendon ends in the ruptured tendon portion within the DIP joint extension position [13].

As the first splint flexes the PIP joint and as the DIP joint is considered the hyperextension position, the ruptured tendon is thought to be sufficiently entwined, more than with the conventional splint at the DIP joint extension position. Considering the tendon repair process, a remarkable proliferation of fiber cells and fibroblasts could be observed at the tendon cross-sectional area at approximately 3 weeks, and tendon tensile strength increased [14]. At this time, the splint was changed to a conventional splint at the DIP joint extension position. This change allowed exercise of the PIP joint, and thereby, is thought to have minimized the PIP joint contracture.

Because case 1 had a severe terminal tendon injury, the lateral band and oblique retinacular ligaments were translocated to the dorsal side, presenting a swan-neck deformity [12]. Therefore, in order to sufficiently reposition the terminal tendon, we planned to fix the finger in the DIP joint hyperextended position for 3 weeks to flex the PIP joint and transpose the lateral band and oblique retinacular ligaments to the volar side.

This suggests that in a case such as case 2, where a bony mallet finger is classified as type IIA or lower according to the Wehbé and Schneider classification system [7], even if this is an obsolete case, good fixation positions can be preserved and synostosis is possible.

In case 2, radiography showed DIP joint extension only, indicating that repositioning of the bone fragment could be achieved through PIP joint flexion and DIP joint extension. The splint was fixed at that position, 
Table 4. Case 2: Course of treatment (right ring finger).

\begin{tabular}{lccccc}
\hline & 1 week & 2 weeks & 6 weeks & 12 weeks & Outcome \\
\hline DIP Ex/Flex & $-30 / 30$ & $20 /-20$ & $20 / 30$ & $5 / 50$ & Crawford \\
Pain VAS & $8 / 10$ & $5 / 10$ & $0 / 10$ & $0 / 10$ & evaluation criteria \\
Work & Some return & Full return & Full return & Full return & Perfect \\
\hline
\end{tabular}

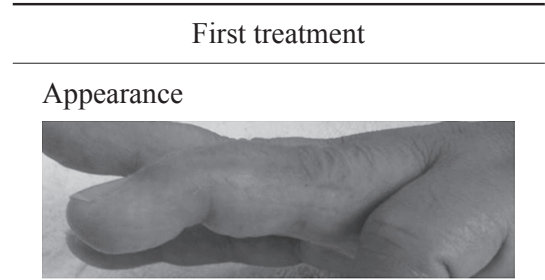

Radiograph

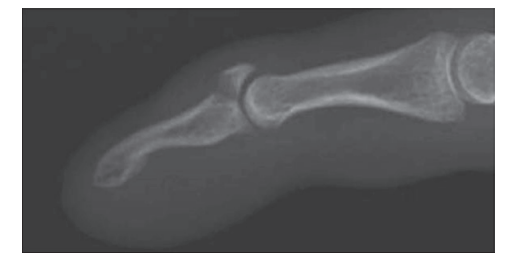

Outcome after 12 weeks

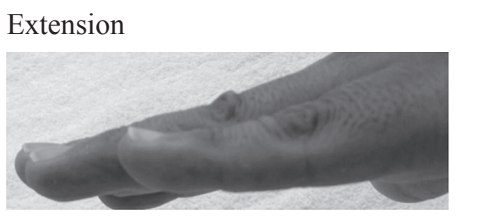

Flexion

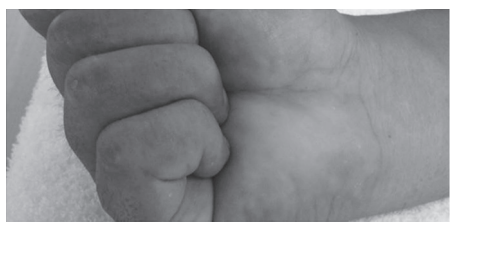

Fig. 3. Case 2: First treatment and outcome after 12 weeks (Right ring finger with bony mallet finger).

which allowed fixation at a position where the terminal tendon was sufficiently loose $[2,4,12]$.

The success of the two-step splinting used in this study was owing to the fact that anatomically, the tension on and looseness of the extensor tendon was maintained in the early stages. Thereafter, switching to a splint that fixed the minimum amount of the digit prevented excess contraction and led to improvement of ROM. For the creation of the first splint, it is important to correctly understand the characteristics of thermoplastics and be familiar with the materials. Moreover, care in the casting is important to ensure that no pressure is applied at the sides of the finger or at the dorsal side of the DIP joint. To increase the possibility of successful fixation of the splint for a continuous period of $\geq 6$ weeks, the splint should be custom-fit for each patient. Custom-made, two-step splinting is thus thought to be effective. The splinting therapy that we used, with its two splinting methods, is thought to have enabled fixation and ROM acquisition that were in tandem with the tendon healing process. In the future, we will accumulate more cases of using this fixation method and investigate, for example, the differences between the two splinting methods and fixation of the DIP joint only, as well as the application of our splinting method to residual mallet finger. This treatment hints at the effectiveness of conservative therapy in the treatment of swan neck deformities and bony mallet finger.

In the future, we would like to continue our study of this treatment but with larger numbers of subjects and with attention paid to splint-related complications such as dorsal skin maceration and necrosis [15]. We would also like to compare conventional treatments with the two-step splint method. Finally, we would like to investigate the applicability of our treatment method to obsolete cases of tendinous and bony mallet fingers.

\section{References}

[1] Clayton RA, Court-Brown CM. The epidemiology of musculoskeletal tendinous and ligamentous injuries. Injury. 2008; 39: 1338-44.

[2] Handoll HH, Vaghela MV. Interventions for treating mallet finger injuries. Cochrane Database Syst Rev. 2004; $1-25$.

[3] Abouna JM. Splint for mallet-finger. Br Med J. 1965; 13: 444.

[4] Cheung JP, Fung B, Ip WY. Review on mallet finger treatment. Hand Surg. 2012; 17: 439-47.

[5] Stack HE. A modified splint for mallet finger. J Hand Surg Br. 1989; 11: 83-9.

[6] Wilson SW, Khoo CT. The Mexican hat splint-a new splint for the treatment of closed mallet finger. J Hand Surg Br. 2001; 26: 488-9.

[7] Wehbé MA, Schneider LH. Mallet fractures. J Bone Joint 
Surg Am. 1984; 66: 658-69.

[8] Evans RB. Therapeutic management of extensor tendon injuries. Hand Clin. 1986; 2: 157-69.

[9] O'Connor JF. Mallet finger. Can Fam Physician. 1997; 43: $1725-6$.

[10] Pike J, Mulpuri K, Metzger M, Ng G, Wells N, Goetz T. Blinded, prospective, randomized clinical trial comparing volar, dorsal, and custom thermoplastic splinting in treatment of acute mallet finger. J Hand Surg Am. 2010; 35: 580-8.

[11] O’Brien LJ, Bailey MJ. Single blind, prospective, randomized controlled trial comparing dorsal aluminum and custom thermoplastic splints to stack splint for acute mallet finger. Arch Phys Med Rehabil. 2011; 92: 191-8.
[12] Harris C Jr, Rutledge GL Jr. The functional anatomy of the extensor mechanism of the finger. J Bone Joint Surg Am. 1972; 54: 713-26.

[13] Schweitzer TP, Rayan GM. The terminal tendon of the digital extensor mechanism: Part II, kinematic study. J Hand Surg Am. 2004; 29: 903-8.

[14] Peacock EE Jr. Biological Principles in the healing of long tendons. Surg Clin North Am. 1965; 45: 461-76.

[15] Rayan GM, Mullins PT. Skin necrosis complicating mallet finger splinting and vascularity of the distal interphalangeal joint overlying skin. J Hand Surg Am. 1987; 12: $548-52$. 